\title{
Impact of a Multidisciplinary Team Approach on Extracorporeal Circulatory Life Support-Bridged Heart Transplantation
}

\author{
Jae Jun Lee, M.D., Young Su Kim, M.D., Suryeun Chung, M.D., Dong Seop Jeong, M.D., Ph.D., Ji-Hyuk Yang, M.D., Ph.D., \\ Kiick Sung, M.D., Ph.D., Wook Sung Kim, M.D., Ph.D., Tae-Gook Jun, M.D., Ph.D., Yang Hyun Cho, M.D., Ph.D. \\ Department of Thoracic and Cardiovascular Surgery, Samsung Medical Center, Sungkyunkwan University School of Medicine, Seoul, Korea
}

\section{ARTICLE INFO}

Received September 16, 2020

Revised November 6, 2020

Accepted November 11, 2020

Corresponding author

Yang Hyun Cho

Tel 82-2-3410-1677

Fax 82-2-3410-6986

E-mail yanghyun.cho@samsung.com

ORCID

https://orcid.org/0000-0003-1685-3641
Background: The number of heart transplantations $(\mathrm{HTX})$ is increasing annually. Due to advances in medical and surgical support, the outcomes of HTx are also improving. Extracorporeal circulatory life support (ECLS) provides patients with decompensated heart failure a chance to undergo HTx. A medical approach involving collaboration among experienced experts in different fields should improve the outcomes and prognosis of ECLSbridged HTx.

Methods: From December 2003 to December 2018, 1,465 patients received ECLS at Samsung Medical Center. We excluded patients who had not undergone HTx or underwent repeated transplantations. Patients younger than 18 years were excluded. We also excluded patients who received an implantable durable left ventricular assist device before HTx. In total, 91 patients were included in this study. A multidisciplinary team approach began in March 2013 at our hospital. We divided the patients into 2 groups depending on whether they were treated before or after implementation of the team approach.

Results: The 30-day mortality rate was significantly higher in the pre-ECLS team group than in the post-ECLS team group ( $n=5,18.5 \%$ vs. $n=2,3.1 \% ; p=0.023$ ). The 1 -year survival rate was better in the post-ECLS team group than in the pre-ECLS team group $(n=57$, $89.1 \%$ vs. $n=19,70.4 \% ; p=0.023$ ).

Conclusion: We found that implementing a multidisciplinary team approach improved the outcomes of ECLS-bridged HTx. Team-based care should be adapted at HTx centers that perform high-risk HTx.

Keywords: Heart transplantation, Extracorporeal circulation, Extracorporeal membrane oxygenation, Patient care team, Multidisciplinary team approach

\section{Introduction}

After the first heart transplantation (HTx) in Korea in 1992, the number of cases of HTx has increased and their outcomes have been favorable [1]. According to a Korean Organ Transplant Registry report, 184 cases of HTx were performed in 2017 [2]. In a recent study, the 1-year survival rate after HTx in Korea was reported to be 90\% [3]. However, in recent years, there have been more elderly recipients and patients with multiple comorbidities than in the early years of HTx in Korea. Furthermore, the use of extracorporeal life support (ECLS) as a bridge to HTx has exponentially increased, owing to the donor shortage and the popularity of ECLS [3-5]. However, the outcomes of ECLS- bridged HTx have been reported to be poorer than those of HTx without ECLS [6].

There are very few reports on the favorable impact of a multidisciplinary team approach in ECLS; therefore, we described the results of applying such an approach in our program $[7,8]$. We hypothesized that the presence of a multidisciplinary team dedicated to ECLS management would contribute to better outcomes in patients with heart failure. In addition, multidisciplinary team management may improve the outcomes and prognosis of patients with ECLSbridged HTx. 


\section{Methods}

\section{Study population}

From December 2003 to December 2018, 1,465 patients received ECLS at Samsung Medical Center (Fig. 1). Only 104 patients on ECLS underwent HTx. We excluded patients who had not undergone HTx or underwent repeated transplantations. Patients younger than 18 years were excluded. We also excluded 4 patients who had undergone durable left ventricular assist device (LVAD) implantation before HTx, since conversion of ECLS to an implantable LVAD followed by HTx was only conducted after the ECLS team was established. Finally, 91 patients were selected for the study. The patients scheduled to undergo transplantation were on a waiting list for HTx administered by the Korean Network for Organ Sharing (KONOS).

In March 2013, we involved a dedicated cardiologist and cardiac surgeon in the care of the patients on ECLS. Thus, the multidisciplinary team approach began in March 2013. We divided the patients into 2 groups, depending on whether they were treated before or after the team approach was implemented.

This retrospective study was approved by the Institutional Review Board of Sungkyunkwan University (IRB approval no., 202009115001). The requirement for patient consent was waived owing to the retrospective nature of this study.

\section{Extracorporeal life support organization and outcomes}

Before the team approach, patients on ECLS were treated separately by cardiovascular surgeons, cardiologists and other consultant physicians. However, after a multidisciplinary ECLS team was established, all the patients on ECLS at our center were under its care. The team consisted of cardiac surgeons, cardiologists, pulmonologists, neurologists, perfusionists, ECLS coordinators, and an intensive care unit (ICU) team. All team members were involved in patient selection, management, HTx listing, recipient selection, and postoperative care (Fig. 2). Thus, the study sub-
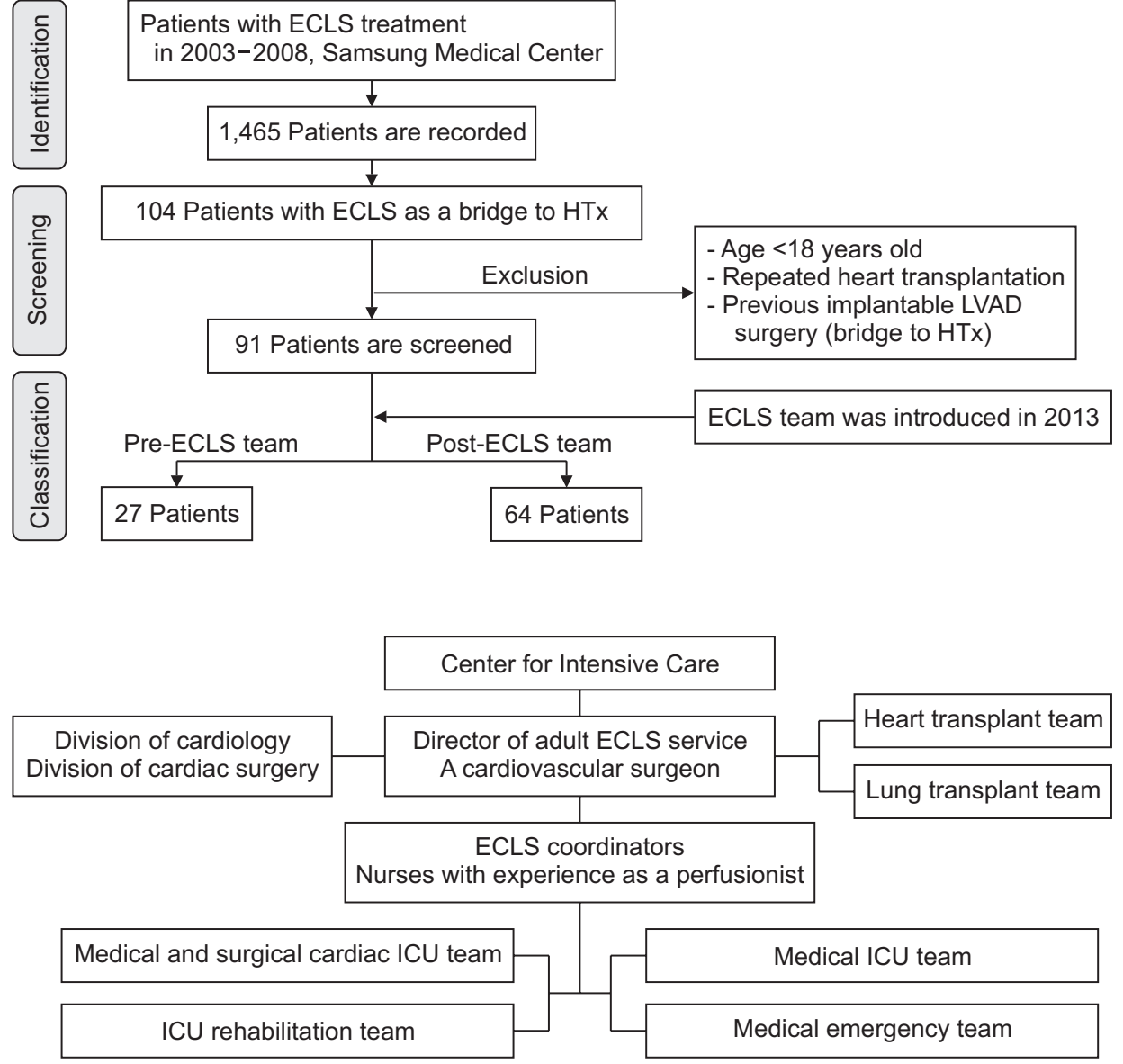

Fig. 1. Flow chart of patient selection. ECLS, extracorporeal circulatory life support; HTx, heart transplantation; LVAD, left ventricular assist device.
Fig. 2. Composition of the multidisciplinary ECLS team. ECLS, extracorporeal circulatory life support; ICU, intensive care unit. 
jects were divided into 2 groups: the pre-ECLS team group and post-ECLS team group. The primary outcome was 30 day mortality and the secondary outcome was 1-year survival.

\section{Statistical analysis}

Categorical variables are presented as numbers and percentages. Continuous variables are presented as means. Data were compared using the Mann-Whitney U-test for continuous variables and the chi-square test or Fisher exact test for categorical variables. Variables with p-values of $<0.2$ in the univariate Cox regression analysis, along with variables that were considered clinically relevant, were entered into the multivariate Cox logistic regression model. We used multivariate logistic regression with the backward stepwise method to assess independent predictors of 30 day mortality. All p-values $<0.05$ were considered to indicate statistical significance. The statistical tool IBM SPSS for Windows ver. 25.0 (IBM Corp., Armonk, NY, USA) was used for analysis.

\section{Results}

\section{Baseline characteristics of patients}

The baseline characteristics of the patients are shown in Table 1 . The mean age of the recipients in the pre-ECLS team group was lower than that of those in the post-ECLS team group $(43.3 \pm 18.1$ years versus $51.2 \pm 12.4$ years, $\mathrm{p}=0.035$ ). The preoperative ECLS duration was significantly longer in the post-ECLS team group than in the preECLS team group $(18.1 \pm 21.5$ days versus $10.9 \pm 10.8$ days, $\mathrm{p}=0.020$ ). The left ventricular ejection fraction was worse in the post-ECLS team group than in the pre-ECLS team group $(27.1 \% \pm 14.3 \%$ versus $20.1 \% \pm 12.1 \%, p=0.006)$. The Sequential Organ Failure Assessment (SOFA) score was also significantly lower in the post-ECLS team group than in the pre-ECLS team group $(12.1 \pm 3.0$ versus $13.5 \pm 3.2$, $\mathrm{p}=0.028)$. Sex, $\mathrm{ABO}$ incompatibility, and underlying diseases were not different between the 2 groups.

\section{Clinical outcomes and predictors of mortality}

The outcomes of HTx with ECLS are shown in Table 2. The postoperative ICU stay and total ICU stay were longer in the post-ECLS team group $(24.2 \pm 22.6$ days and $43.6 \pm 30.6$ days, respectively) than in the pre-ECLS team

Table 1. Patients' demographic and baseline clinical characteristics

\begin{tabular}{|c|c|c|c|}
\hline Characteristic & Pre-ECLS team $(n=27)$ & Post-ECLS team $(n=64)$ & p-value \\
\hline Recipient age $^{\text {a) }}(y r)$ & $43.3 \pm 18.1$ & $51.2 \pm 12.4$ & 0.035 \\
\hline Recipient sex (male) & $18(66.7)$ & $44(68.8)$ & 0.846 \\
\hline Donor age $(y r)$ & $40.1 \pm 12.2$ & $41.1 \pm 11.1$ & 0.402 \\
\hline Donor sex (male) & $23(31.9)$ & $49(76.6)$ & 0.355 \\
\hline $\mathrm{ABO}$ incompatibility & $22(81.5)$ & $45(70.3)$ & 0.269 \\
\hline Previous open heart surgery & $6(22.2)$ & $20(31.3)$ & 0.384 \\
\hline Hypertension & $6(22.2)$ & $23(35.9)$ & 0.200 \\
\hline Cerebrovascular accident & $3(11.1)$ & $3(4.7)$ & 0.357 \\
\hline Diabetes mellitus & $5(18.5)$ & $19(29.7)$ & 0.269 \\
\hline Overall hospital days (day) & $143.8 \pm 471$ & $73.3 \pm 84.2$ & 0.480 \\
\hline Preoperative ECLS duration ${ }^{\text {a) }}$ (day) & $10.9 \pm 10.8$ & $18.1 \pm 21.5$ & 0.020 \\
\hline Preoperative intensive care unit stay (day) & $15.5 \pm 13.2$ & $19.3 \pm 21.9$ & 0.327 \\
\hline Left ventricle ejection fraction ${ }^{a)}(\%)$ & $27.1 \pm 14.3$ & $20.1 \pm 12.1$ & 0.006 \\
\hline Preoperative coronary intervention & $2(7.4)$ & $15(23.4)$ & 0.073 \\
\hline Preoperative serum platelet count $\left(\times 10^{3} / \mu \mathrm{L}\right)$ & $102.9 \pm 48.4$ & $110.7 \pm 58.1$ & 0.300 \\
\hline Preoperative serum albumin $(\mathrm{g} / \mathrm{dL})$ & $3.0 \pm 0.4$ & $3.1 \pm 0.4$ & 0.365 \\
\hline Preoperative serum total bilirubin (mg/dL) & $7.2 \pm 9.2$ & $6.0 \pm 9.2$ & 0.149 \\
\hline Preoperative continuous renal replacement treatment & $7(25.9)$ & $19(30.6)$ & 0.653 \\
\hline Sequential Organ Failure Assessment score ${ }^{\mathrm{a})}$ & $13.5 \pm 3.2$ & $12.1 \pm 3.0$ & 0.028 \\
\hline
\end{tabular}

Values are presented as mean \pm standard deviation or number (\%).

ECLS, extracorporeal life support.

${ }^{a}$ Variables with statistical significance. 
Table 2. Outcomes of heart transplantation with ECLS

\begin{tabular}{lccc}
\hline \multicolumn{1}{c}{ Variable } & Pre-ECLS team $(\mathrm{n}=27)$ & Post-ECLS team $(\mathrm{n}=64)$ & $\mathrm{p}$-value \\
\hline Postoperative ICU stay (day) & $20.2 \pm 20.7$ & $24.2 \pm 22.6$ & 0.130 \\
Total ICU stay (day) & $35.8 \pm 25.8$ & $43.6 \pm 30.6$ & 0.093 \\
Postoperative ECLS & $9(33.3)$ & $12(18.8)$ & 0.131 \\
Postoperative ECLS duration (hr) & $32.8 \pm 55.3$ & $15.3 \pm 40.4$ & 0.052 \\
Hyperacute rejection & $1(3.7)$ & $2(3.1)$ & 1 \\
Acute rejection & $8(29.6)$ & $11(17.2)$ & 0.182 \\
30-Day mortality & $5(18.5)$ & $2(3.1)$ & 0.023 \\
Cause of death & $1(20)$ & $2(100)$ & 0 \\
$\quad$ Hyperacute rejection & $2(40)$ & 0 & \\
Septic shock & $1(20)$ & 0 & \\
Postoperative bleeding & $1(20)$ & & \\
Upper gastrointestinal bleeding & & & \\
\hline
\end{tabular}

Values are presented as mean \pm standard deviation or number (\%).

ECLS, extracorporeal life support; ICU, intensive care unit.

Table 3. Operative data

\begin{tabular}{lccr}
\hline \multicolumn{1}{c}{ Variable } & Pre-ECLS team $(\mathrm{n}=27)$ & Post-ECLS team $(\mathrm{n}=64)$ & $\mathrm{p}$-value \\
\hline Operation time $(\mathrm{min})$ & $441.0 \pm 65.9$ & $467.5 \pm 102$ & 0.526 \\
Cardiopulmonary bypass time $(\mathrm{min})$ & $172.0 \pm 38.5$ & $144.0 \pm 38.0$ & $<0.001$ \\
Total ischemic time $(\mathrm{min})$ & $188.4 \pm 67.1$ & $195.5 \pm 59.9$ & 0.520 \\
Major bleedinga) $^{\text {a) }}$ & $7(25.9)$ & $7(10.9)$ & 0.109 \\
\hline
\end{tabular}

Values are presented as mean \pm standard deviation or number (\%).

ECLS, extracorporeal life support.

a)Major bleeding was defined as requiring an operation for bleeding control.

group (20.2 \pm 20.7 days and $35.8 \pm 25.8$ days, respectively). However, this difference was not statistically significant.

The 30-day mortality rate was significantly higher in the pre-ECLS team group than in the post-ECLS team group $(\mathrm{n}=5,18.5 \%$ versus $\mathrm{n}=2,3.1 \% ; \mathrm{p}=0.023)$. Postoperative ECLS implementation was more common and lasted longer in the pre-ECLS team group than in the post-ECLS team group $(9$ [33.3\%] versus 12 [18.8\%], $\mathrm{p}=0.131 ; 32.8 \pm 55.3$ hours versus $15.3 \pm 40.4$ hours, $\mathrm{p}=0.052)$, but these differences were not statistically significant. Operation time and ischemic time were similar in both groups. Cardiopulmonary bypass time was longer in the pre-ECLS team group than in the post-ECLS team group $(172.0 \pm 38.5$ minutes versus $144.0 \pm 38.0$ minutes, $\mathrm{p}<0.001$ ) (Table 3 ).

The Kaplan-Meier curve log-rank test also showed that the 1-year survival rate was significantly higher in the post-ECLS team group than in the pre-ECLS team group $(\mathrm{n}=57,89.1 \%$ versus $\mathrm{n}=19,70.4 \%$; $\mathrm{p}=0.023)$ (Fig. 3).

In univariate Cox regression analysis, the ECLS team approach (odds ratio [OR], 0.327; 95\% confidence interval [CI], $0.118-0.902 ; \mathrm{p}=0.031)$ was a positive predictor of 1-year survival, while total bilirubin (OR, 1.058; 95\% CI, $1.027-1.090 ; \mathrm{p}<0.001)$ and the SOFA score (OR, 1.421; $95 \%$

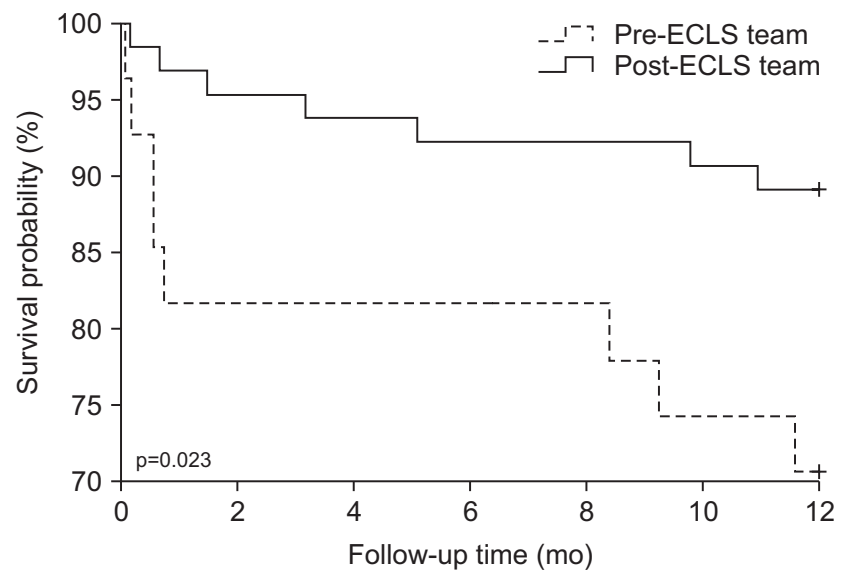

Fig. 3. Kaplan-Meier curve and log rank test of the pre- and postECLS team groups. One-year survival was better in the post-ECLS team group than in the pre-ECLS team group $(n=57,89.1 \%$ vs. $n=19,70.4 \% ; p=0.023)$. ECLS, extracorporeal circulatory life support.

CI, 1.187-1.702; $\mathrm{p}<0.001)$ were negative predictors. In the multivariate Cox regression analysis, the ECLS team approach (OR, 0.226; 95\% CI, 0.079-0.647; $\mathrm{p}=0.006)$, total bilirubin (OR, 1.072; 95\% CI, 1.036-1.109; $\mathrm{p}<0.001)$, and 
Table 4. Univariate and multivariate Cox proportional hazard ratios for 1-year mortality

\begin{tabular}{|c|c|c|c|c|}
\hline \multirow{2}{*}{ Variable } & \multicolumn{2}{|c|}{ Univariate analysis } & \multicolumn{2}{|c|}{ Multivariate analysis } \\
\hline & OR $(95 \% \mathrm{Cl})$ & p-value & OR $(95 \% \mathrm{Cl})$ & p-value \\
\hline Post-ECLS team & $0.327(0.118-0.902)$ & 0.031 & $0.226(0.079-0.647)$ & 0.006 \\
\hline Recipient age & $1.032(0.991-1.075)$ & 0.127 & $1.048(1.008-1.089)$ & 0.017 \\
\hline Recipient sex & $1.480(0.527-4.157)$ & 0.457 & & \\
\hline Donor age & $0.988(0.947-1.030)$ & 0.560 & & \\
\hline Donor sex & $1.899(0.649-5.556)$ & 0.242 & & \\
\hline $\mathrm{ABO}$ incompatibility & $0.991(0.315-3.112)$ & 0.987 & & \\
\hline Previous open heart surgery & $1.334(0.456-3.904)$ & 0.599 & & \\
\hline Hypertension & $1.070(0.366-3.130)$ & 0.902 & & \\
\hline Cerebrovascular accident & $0.044(0.000-59.765)$ & 0.482 & & \\
\hline Diabetes mellitus & $0.410(0.092-1.815)$ & 0.240 & & \\
\hline Preop ECLS duration & $0.962(0.908-1.020)$ & 0.194 & & \\
\hline Preop intensive care unit stay & $0.966(0.916-1.018)$ & 0.199 & & \\
\hline Left ventricular ejection fraction & $1.014(0.983-1.047)$ & 0.377 & & \\
\hline Preop coronary intervention & $0.628(0.142-2.782)$ & 0.540 & & \\
\hline Preop serum platelet & $0.995(0.984-1.006)$ & 0.394 & & \\
\hline Preop serum albumin & $0.598(0.181-1.976)$ & 0.399 & & \\
\hline Preop serum total bilirubin & $1.058(1.027-1.090)$ & $<0.001$ & $1.072(1.036-1.109)$ & $<0.001$ \\
\hline Preop continuous renal replacement treatment & $1.718(0.611-4.828)$ & 0.305 & & \\
\hline Sequential Organ Failure Assessment score & $1.421(1.187-1.702)$ & $<0.001$ & & \\
\hline
\end{tabular}

OR, odds ratio; $\mathrm{Cl}$, confidence interval; ECLS, extracorporeal life support; Preop, preoperative.

recipient age (OR, 1.048; 95\% CI, 1.008-1.089; $\mathrm{p}=0.017$ ) were independent predictors of 1-year survival (Table 4).

\section{Discussion}

ECLS is commonly used for the treatment of cardiogenic shock. Intensive care with ECLS is inevitable in patients with acute refractory heart failure. However, no firm consensus exists regarding the appropriate timing of ECLS implementation for cardiogenic shock. Thus, an early recognition of signs of deterioration, just before profound shock status, is critical for patient selection, early management, and intervention timing [9]. Furthermore, the duration of ECLS is closely related to various complications. Maintaining ECLS for a longer time increases the risk of inflammatory reactions, infections, neurological complications, and other complications [10-13]. Because of these complex conditions, the current trend is to manage patients on ECLS using a multidisciplinary team approach. A professional team with accumulated experience may provide an advanced quality of management and outcomes for patients on ECLS bridged to HTx $[14,15]$. Our study showed that patients in the post-ECLS team group were older than those in the pre-ECLS team group. The age-related criteria for HTx were recently extended and HTx prioritization was revised by the KONOS in 2018. Therefore, we actively performed transplantation surgery in such cases. Moreover, the total mean duration of ECLS before transplantation was significantly longer in the post-ECLS team than in the pre-ECLS team. The longest duration of preoperative ECLS management was 48 days and 126 days in the pre-ECLS and post-ECLS team groups, respectively. The multidisciplinary approach of the ECLS team allows extension of the transplantation opportunity for patient selection and effective management.

Early mortality was significantly higher in the pre-ECLS team group than in the post-ECLS team group. Postoperative septic shock and bleeding could be related to the patients' preoperative condition, as the average SOFA score was lower in the post-ECLS team group than in the preECLS team group. However, the cardiopulmonary bypass time was shorter in the post-ECLS team group than in the pre-ECLS team group, which affected the likelihood of postoperative bleeding-related complications. One case of hyperacute rejection in post-ECLS team group was demonstrated by heart biopsy. The others were diagnosed by clinical assessment, and the heart biopsy could not demonstrate graft rejection.

We tried to determine the factors affecting 1-year survival by Cox regression analysis. In multivariate analysis, older age of the recipients was associated with a poor 1-year survival rate [16]. Two other factors, namely, low preopera- 
tive serum total bilirubin and the ECLS team approach, were statistically significant predictors of 1-year survival. Because of the congestive physiology in heart failure, hepatic dysfunction is associated with the progression of heart failure. It is well known that hepatic dysfunction before and after transplantation results in a poor prognosis [17]. In cardiogenic shock, multi-organ dysfunction could further deteriorate and be fatal unless the condition is resolved. Therefore, early restoration of cardiac function following improvement in organ perfusion is critically necessary for favorable outcomes. Likewise, the preoperative SOFA score, which represents patients' general condition, was lower in the post-ECLS team group than in the preECLS team group. Preoperative serum bilirubin levels were also lower in the post-ECLS team group than in the preECLS team group, but this difference did not show statistical significance. As shown in Table 2, the differences in postoperative ECLS application and duration between the pre-ECLS team group and the post-ECLS team group may imply that patients had different preoperative conditions and postoperative management.

Before the ECLS team was established, the management of patients with cardiogenic shock differed depending on the medical team. Because the accumulation of experience with ECLS is concentrated in a specific team, the ECLS team will manage patients consistently and efficiently. Therefore, decisions related to time-critical patients can effectively be made using a multidisciplinary team approach.

During the study period, there was 1 major change related to the study. Specifically, since 2017, antifungal prophylaxis has been administered to ECLS patients undergoing HTx.

Our study has some limitations. First, all data for this study were collected retrospectively. Second, the population size was too small for standardization. Third, the findings were affected by selection bias regarding patients' basic characteristics. There may be some differences in characteristics between patients with acute or chronic decompensated heart failure; however, we could not reflect these possible differences in our study. Furthermore, the time interval between the pre-ECLS team and post-ECLS team groups was up to 15 years, and the overall medical environment, which has also developed over this period, may have contributed to the improved survival rate. Since patients who underwent conversion to LVAD implantation from ECLS were excluded from this study, further studies should investigate the outcomes achieved by the ECLS team in patients with LVAD bridging to HTx.

In conclusion, we found that a multidisciplinary team approach improved the outcomes of ECLS-bridged HTx. Team-based care should be adapted at HTx centers that perform high-risk HTx.

\section{Conflict of interest}

No potential conflict of interest relevant to this article was reported.

\section{ORCID}

Jae Jun Lee: https://orcid.org/0000-0003-0466-7630

Young Su Kim: https://orcid.org/0000-0003-2923-3732

Suryeun Chung: https://orcid.org/0000-0002-9619-0640

Dong Seop Jeong: https://orcid.org/0000-0002-6947-8403

Ji-Hyuk Yang: https://orcid.org/0000-0002-7349-6778

Kiick Sung: https://orcid.org/0000-0003-0768-9587

Wook Sung Kim: https://orcid.org/0000-0001-7808-3385

Tae-Gook Jun: https://orcid.org/0000-0002-5161-4665

Yang Hyun Cho: https://orcid.org/0000-0003-1685-3641

\section{References}

1. Lee HY, Jeon ES, Kang SM, Kim JJ. Initial report of the Korean Organ Transplant Registry (KOTRY): heart transplantation. Korean Circ J 2017;47:868-76.

2. Kim IC, Youn JC, Kobashigawa JA. The past, present and future of heart transplantation. Korean Circ J 2018;48:565-90.

3. Kim D, Choi JO, Oh J, et al. The Korean Organ Transplant Registry (KOTRY): second official adult heart transplant report. Korean Circ J 2019;49:724-37.

4. Youn JC, Han S, Ryu KH. Temporal trends of hospitalized patients with heart failure in Korea. Korean Circ J 2017;47:16-24.

5. Youn JC, Kim IC, Park NH, Kim H. Increased risk with older donor age and more frequent pre-transplant ECMO: the second official KOTRY report. Korean Circ J 2019;49:738-41.

6. Fukuhara S, Takeda K, Kurlansky PA, Naka Y, Takayama H. Extracorporeal membrane oxygenation as a direct bridge to heart transplantation in adults. J Thorac Cardiovasc Surg 2018;155:1607-18.

7. Hong D, Choi KH, Cho YH, et al. Multidisciplinary team approach in acute myocardial infarction patients undergoing veno-arterial extracorporeal membrane oxygenation. Ann Intensive Care 2020;10:83.

8. Na SJ, Chung CR, Choi HJ, et al. The effect of multidisciplinary extracorporeal membrane oxygenation team on clinical outcomes in patients with severe acute respiratory failure. Ann Intensive Care 2018;8:31.

9. Reyentovich A, Barghash MH, Hochman JS. Management of refractory cardiogenic shock. Nat Rev Cardiol 2016;13:481-92.

10. Millar JE, Fanning JP, McDonald CI, McAuley DF, Fraser JF. The 
inflammatory response to extracorporeal membrane oxygenation (ECMO): a review of the pathophysiology. Crit Care 2016;20:387.

11. Biffi S, Di Bella S, Scaravilli V, et al. Infections during extracorporeal membrane oxygenation: epidemiology, risk factors, pathogenesis and prevention. Int J Antimicrob Agents 2017;50:9-16.

12. Xie A, Lo P, Yan TD, Forrest P. Neurologic complications of extracorporeal membrane oxygenation: a review. J Cardiothorac Vasc Anesth 2017;31:1836-46.

13. Ouweneel DM, Schotborgh JV, Limpens J, et al. Extracorporeal life support during cardiac arrest and cardiogenic shock: a systematic review and meta-analysis. Intensive Care Med 2016;42:1922-34.

14. Dalia AA, Ortoleva J, Fiedler A, Villavicencio M, Shelton K, Cudemus GD. Extracorporeal membrane oxygenation is a team sport: in- stitutional survival benefits of a formalized ECMO team. J Cardiothorac Vasc Anesth 2019;33:902-7.

15. Komindr A, Abe R, Tateishi Y, et al. Establishing extracorporeal membrane oxygenation team increased number of patients and improved data recording. J Intensive Care 2019;7:11.

16. Foroutan F, Alba AC, Guyatt G, et al. Predictors of 1-year mortality in heart transplant recipients: a systematic review and meta-analysis. Heart 2018;104:151-60.

17. Chokshi A, Cheema FH, Schaefle KJ, et al. Hepatic dysfunction and survival after orthotopic heart transplantation: application of the MELD scoring system for outcome prediction. J Heart Lung Transplant 2012;31:591-600. 